Med Klin Intensivmed Notfmed 2019 • 114:504-508 https://doi.org/10.1007/s00063-019-0596-1

Eingegangen: 5. Februar 2019

Angenommen: 7. Februar 2019

Online publiziert: 7. August 2019

(c) Springer Medizin Verlag GmbH, ein Teil von

Springer Nature 2019

\section{Redaktion}

G. Michels, Köln

\section{Hintergrund}

Die Lungensonographie (LUS) hat sich im Vergleich zu anderen häufig angewandten Ultraschallverfahren, wie die Echokardiographie bzw. die AbdomenGefäß- und gynäkologische Sonographie, relativ spät entwickelt, weil an der gesunden Lunge über das Pleuragleiten hinaus sonographisch nur Artefakte darstellbar sind.

In den letzten 2 Dekaden sind jedoch sehr viele Arbeiten erschienen, die den Wert der LUS besonders in Notfallsituationen belegen. Diese wurden auch in einer intensiven, internationalen Konsensuskonferenz zusammengefasst [1]. Anschließend hat sich ein wahrer Boom an Publikationen zur LUS entwickelt. Unter dem Titel „lung ultrasound“ sind in PubMed gegenwärtig 91.710 Titel gelistet.

》) Die LUS führt in 80-90 \% der Erkrankungen der Lunge oder des Herzens zur Diagnose

Die LUS ist eine symptomorientierte „Point-of-care“-Sonographie (POCUS): Sie ermöglicht eine Bildgebung genau an der Stelle der Beschwerden bzw. dient der Ursachendiagnostik einer Atemnot. Die ersten Fragen lauten: Was ist das Leitsymptom, gibt es Begleitsymptome? Liegen Luftnot bzw. Schmerzen vor oder hat der Patient/die Patientin Fieber? Dabei gibt es oft Überlappungen, die zu bedenken sind (-Abb. 1). Nach Anamnese und klinischer Untersuchung kann die symptomorientierte Sonographie sofort eingesetzt werden und führt in

\title{
Gebhard Mathis
}

Rankweil, Österreich

\section{Einsatz der Lungen- und Pleurasonographie in der Notfall- und Intensivmedizin}

80-90\% der Erkrankungen der Lunge oder des Herzens (z.B. Lungenödem als Ausdruck einer dekompensierten Herzinsuffizienz) zur Diagnose [2].

Eine große italienische Multizenterstudie an 2683 Patienten hat gezeigt, dass in der Notaufnahme mit Einsatz der LUS die Zeit bis zur Diagnose und Therapie 24 min beträgt im Gegensatz zu 186 min bei üblicher Notfalldiagnostik mit anderen bildgebenden Verfahren [3].

Notfallsonographische Konzepte haben die rein fachbezogenen Ultraschallverfahren gesprengt und eine interdisziplinäre Entwicklung eingeleitet. Dies zeigte sich zunächst in der Traumatologie mit dem „focused assessment with sonography for trauma" (FAST), dann erweitert um die Pneumothoraxdiagnostik („,extended“ FAST). Da sich jedoch die meisten Patienten im Notfall mit internistischen Erkrankungen vorstellen, hat sich die Notfallsonographie beträchtlich ausgeweitet, besonders auch auf die Lunge [4].

\section{》) Die Entwicklung portabler Ultraschallsysteme stellt eine Revolution in der Notfallsonographie dar}

Die wirkliche Revolution in der Notfallsonographie fand durch die Entwicklung portabler Ultraschallsysteme statt, die eine bettseitige Untersuchung ubiquitär ermöglichen. Mit einem Schallkopf in der Kitteltasche, der das Bild auf das Smartphone überträgt, wird die Sonographie die Auskultation mit dem Stethoskop ablösen.

Wozu dient dann noch die überflüssige radiologische Bildgebungen, wozu das Ritual der täglichen Röntgenthoraxuntersuchung im Bett auf Intensivstationen? Prinzipiell steht die LUS auf 2 Säulen: (1.) auf der Darstellung von subpleuralen Lungenkonsolidierungen, (2.) auf der Interpretation von Artefakten, die an der Pleura entstehen.

\section{Technische Voraussetzungen}

Für die Thoraxwand und zur Beurteilung der Pleura sind Linearschallsonden mit $5-8 \mathrm{MHz}$ sehr geeignet, wie sie für die Gefäß- oder Schilddrüsensonographie verwendet werden. Es empfiehlt sich eine entsprechende Voreinstellung, die zwischen "muskuloskelettal“ und „Schilddrüse“ liegt. Für tieferliegende Lungenkonsolidierungen reicht die Abdominalsonde. Der Echoschallkopf eignet sich jedoch auch, weswegen der kar-

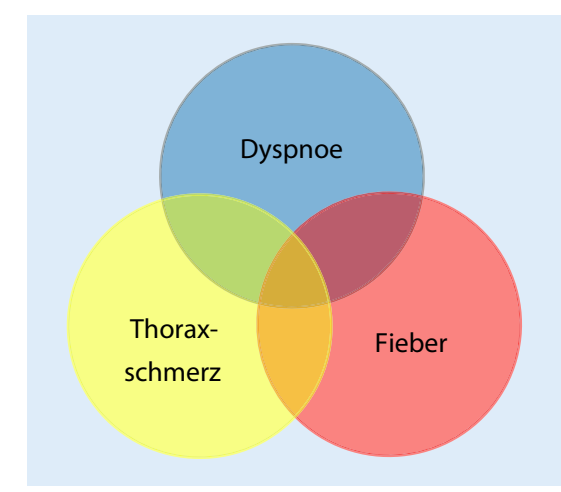

Abb. $1 \Delta$ Die Lungensonographie als symptomorientierte, fokussierte Untersuchung. (Adaptiert nach [2]) 

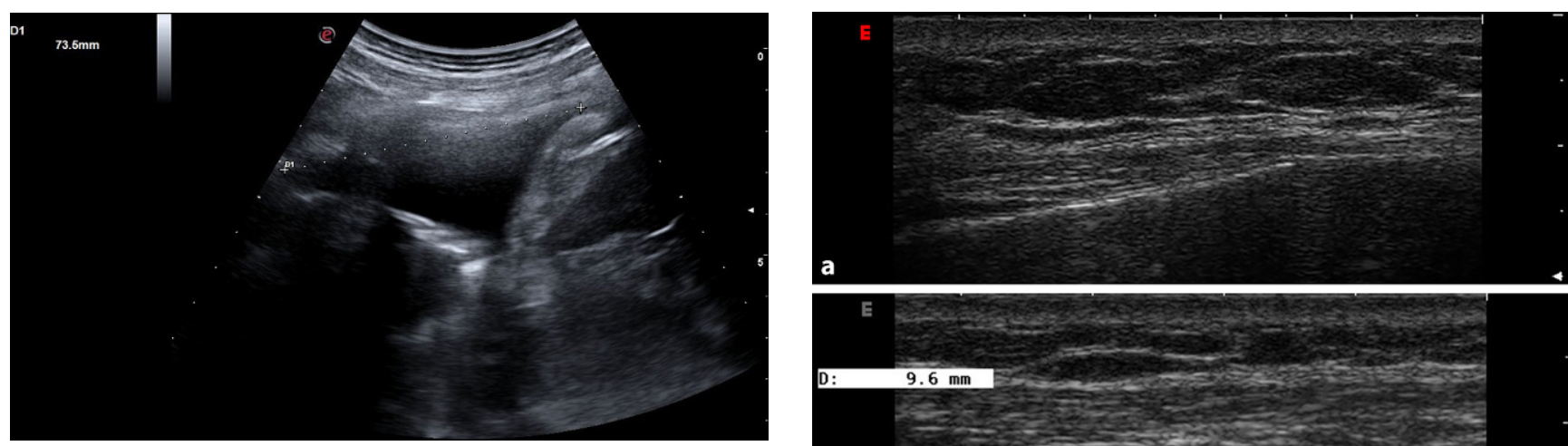

Abb. 2 ム Pleuraergussschätzung beim „sitzenden“ Patienten: $74 \mathrm{~mm} \times 70=$ etwa $500 \mathrm{ml}$

diologisch versierte Untersucher nicht selten die Echokardiographie und die anschließende Lungensonographie mittels Sektorschallkopf durchführt. Wenn man nur eine Sonde an einem portablen Gerät zur Verfügung hat, sollte diese mit $5 \mathrm{MHz}$ arbeiten und mikrokonvex sein, um die Interkostalzwischenräume besser beurteilen zu können [4].

Die Position der Patienten hängt von der Symptomatik bzw. der Fragestellung ab. Der Nachweis eines Pneumothorax oder eines Lungenödems wird idealerweise am liegenden Patienten bzw. in halbsitzender Position erfolgen. Lungenkonsolidierungen lassen sich besser im Sitzen darstellen, zumal diese meisten dorsal lokalisiert sind. Im Notfall können liegende Probanden etwas zur Seite gedreht werden. Im Fall einer akuten Dyspnoe bis Orthopnoe lässt sich der Patient im Sitzen relativ gut sonographisch untersuchen.

\section{Pleuraerguss}

Minimale Ansammlungen von Flüssigkeit im Pleuraraum lassen sich ab etwa $5 \mathrm{ml}$, also bis in den physiologischen Bereich, sonographisch nachweisen. Kardiale Stauungsergüsse sind in aller Regel echolos, solange diese noch nicht punktiert wurden (• Abb. 2). Septen bzw. Septierungen können auf ein Lungenempyem deuten, Knotenbildungen auf dem Zwerchfell sind hoch malignitätsverdächtig [5].

Pleuraergüsse sind so vielgestaltig, dass eine exakte Volumetrie nicht möglich ist. Eine Volumenschätzung ist

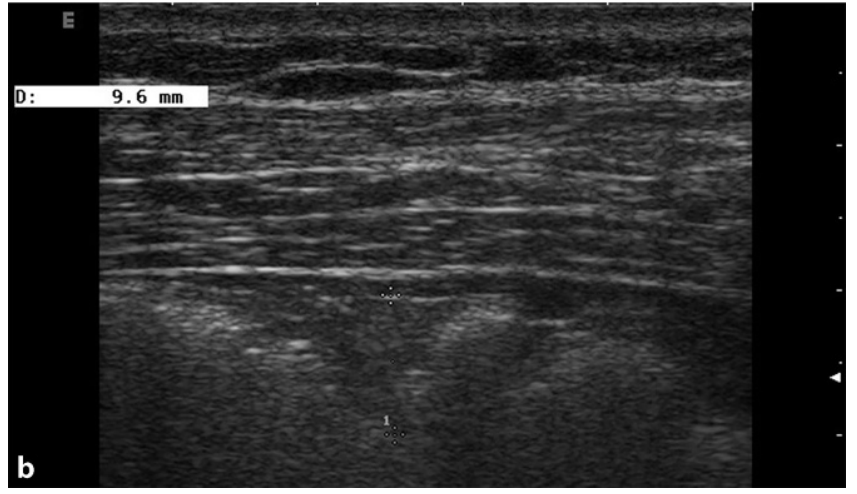

Abb. 3 A Sonographisches Bild einer Rippenfraktur (a) nach Sturz von der Leiter, die in der Röntgendiagnostik nicht darstellbar war. Lungenkontusion mit schmalem Pleuraerguss (b). (Aus [2] mit freundlicher Genehmigung (c) S. Karger AG, Basel)

zielführend, zumal sie in der Frage, ob ein Erguss punktionswürdig ist, gerade im Bereich von $400-600 \mathrm{ml}$ gut zutrifft. Kleine Ergüsse werden im Volumen eher überschätzt, größere hingegen unterschätzt. Beim „liegenden“ oder wegen Atemnot etwas aufgerichteten Patienten (25-30\%) ist eine Messung von der dorsalen Brustwand zum Lungenunterrand in $\mathrm{mm} \times 20$ relativ treffsicher $(\kappa=0,72 ;[6])$. Hier zeigt sich auch eine gute Übereinstimmung mit computertomographisch geführten Pleuraergussschätzungen [7].

\section{Thoraxtrauma}

Bei traumatischen Thoraxverletzungen kann die POCUS oft rasch zu einer Klärung führen. Rippenfrakturen lassen sich mit Stufe, Lücke und einem Reverberationsartefakt („Kaminphänomen") am Schmerzpunkt 2- bis 4-mal häufiger sonographisch darstellen als im Röntgenthoraxbild [8]. An der Brustwand nichttastbare und nichtsichtbare Weichteilverletzungen und Hämatome lassen sich leicht detektieren. Dazu sind weitere Läsionen, wie Hämatothorax (pleurale Flüssigkeit mit tanzenden Binnenechos), Pneumothorax, Perikardtam- ponade und bedrohliche Lungenkontusionen (• Abb. 3), sofort zu entdecken. Beim Thoraxtrauma zeigen sich in $18 \%$ subpleurale Konsolidierungen, die überwacht werden sollten, da diese Patienten noch $48 \mathrm{~h}$ später ein „acute respiratory distress syndrome" (ARDS) entwickeln können. Leichtere Thoraxtraumen zeigen ein fokales interstitielles Syndrom [8].

\section{Pneumothorax}

Ein Pneumothorax, ob spontan oder traumatisch aufgetreten, kann in kurzer Zeit lebensbedrohlich werden.

Sonographische Zeichen eines Pneumothorax sind:

- fehlendes Lungengleiten,

- fehlende B-Linien,

- fehlender Lungenpuls,

- horizontale Reverberationen,

- vorhandene(r) Lungenpunkt(e; [1]).

Der Lungenpuls lässt sich sonographisch in der Power-Doppler-Sonographie leichter nachweisen als im M-Mode. Die Treffsicherheit des Ultraschalls in der Diagnostik des Pneumothorax liegt deutlich über $90 \%$, wenn alle Kriterien berücksichtigt werden. Sie ist deutlich 
besser als die der Röntgenuntersuchung des Thorax und vergleichbar mit der Computertomographie. Die Ausdehnung eines Pneumothorax kann sonographisch nicht beurteilt werden, dazu ist radiologische Bildgebung erforderlich. Ein lebensbedrohlicher Pneumothorax kann jedoch unter LUS-Kontrolle sofort drainiert und in der Entfaltung der Lunge kontrolliert werden [9].

\section{Pleuritis}

Starke, atemabhängige, lokalisierte und stechende Brustschmerzen bestehen bei Pleuritis, weshalb diese Patienten häufig Notaufnahmen besuchen. Ein knarrendes oder reibendes Atemgeräusch ist besonders bei Pleuritis exsudativa mit begleitendem Pleuraerguss durch Auskultation mit dem Stethoskop kaum feststellbar. Sonographisch zeigen sich am Schmerzpunkt Verdickungen und Fragmentierungen der Pleura visceralis mit diskreten subpleuralen Konsolidierungen und vermehrten Kometenschweifartefakten (fokales interstitielles Syndrom), die in der Röntgendiagnostik nicht nachweisbar sind [10].

\section{Interstitielles Syndrom}

Ein Syndrom ist noch keine Diagnose, sondern eine Ansammlung von Symptomen und Befunden, die auf eine bestimmte Krankheit hinweisen kann. Vor über 20 Jahren hat Daniel Lichtenstein gezeigt, dass sich durch vermehrte Kometenschweifarte ein Lungenödem von einer Exazerbation einer chronisch-obstruktive Lungenerkrankung differenzieren lässt [11]. Diese speziellen Kometenschweife werden heute B-Linien genannt. B-Linien sind als diskrete laserähnliche echoreiche Reverberationsartefakte definiert, die an der Pleuralinie entstehen, sich über den ganzen Bildschirm erstrecken und sich synchron mit dem Lungengleiten bewegen (früher: „Kometenschweifartefakte“).

Multiple B-Linien sind das sonographische Zeichen eines interstitiellen Lungensyndroms. Eine positive Region ist durch das Vorhandensein von $\geq 3$ B-Linien im Längsschnitt zwischen 2 Rippen definiert. Idealerweise sollten B-Linien

Med Klin Intensivmed Notfmed 2019 · 114:504-508 https://doi.org/10.1007/s00063-019-0596-1 (c) Springer Medizin Verlag GmbH, ein Teil von Springer Nature 2019

\section{G. Mathis}

\section{Einsatz der Lungen- und Pleurasonographie in der Notfall- und Intensivmedizin}

\section{Zusammenfassung}

Die bettseitige Lungensonographie (LUS) dient in Notaufnahmen und auf Intensivstationen als wertvolles Instrument, um häufige Lungenerkrankungen zu diagnostizieren. Der Verlauf kann kontrolliert und die klinische Strategie geplant werden. Die LUS benötigt wenige Minuten und ist eine brauchbare Ergänzung zur physikalischen Untersuchung. Frakturen der Rippen und des Sternums können gut dargestellt werden. Minimale pleurale Flüssigkeitsansammlungen (Erguss, Hämatothorax) werden detektiert. Mittels LUS kann zwischen einem Lungenödem und einer Exazerbation einer chronischobstruktiven Lungenerkrankung differenziert werden. Entzündungen, wie Pleuritis und Pneumonie, sind besser darstellbar als im Röntgenthoraxbild, sodass LUS bei ambulant erworbener Pneumonie die Röntgenuntersuchung ersetzen könnte. Bei ventilatorassoziierten Pneumonien oder Atelektasen können diese dargestellt werden und es kann die Wiederbelüftung gut kontrolliert werden. Eine Herz-LungenBeinvenen-Triple-Sonographie entsprechend dem klinischen Status hilft, die Diagnose der Lungenembolie zu verbessern. Diese sollte ein Instrument für viele Ärzte sein, speziell in Notaufnahmen.

Schlüsselwörter

Diagnostischer Ultraschall · Pleuraerguss - Pneumonie - Thrombembolie . Lungenverletzungen

\section{Use of lung and pleural ultrasonography in emergency and intensive care medicine}

\begin{abstract}
Bedside lung ultrasound (LUS) in emergency rooms and intensive care units can serve as a tool to diagnose common lung pathologies, monitor their course and guide clinical management. LUS requires only a few minutes and is a useful extension of the physical examination. Fractures of the ribs as well as the sternum are seen well on ultrasound. Minute pleural fluids (effusion, hemtothorax) are detectable. LUS is able to detect the sound of lung water and thus to differentiate a cardiogenic pulmonary edema from chronic obstructive lung disease. Inflammatory lung diseases such as pleuritis and pneumonia are better seen than on chest $X$-ray. LUS should replace chest X-ray in the
\end{abstract}

diagnosis of ambulant acquired pneumonia. In ventilator-associated pneumonia and atelectasis, LUS measures the presence of lung consolidation as well as dynamic changes und reventilation. A heart-lungvessel integrated triple ultrasonography according to clinical findings can help with the diagnosis of pulmonary embolism and should be a necessary weapon for the physicians, especially in emergency departments.

\section{Keywords}

Diagnostic ultrasound · Pleural effusion

Pneumonia - Thromboembolism · Lung injuries in 8 Regionen präsent sein, doch auch ein rascher Blick auf 2 Regionen kann ausreichen [1]. Eine Untersuchung der vorderen oberen Quadraten sowie dorsobasal ist ausreichend, zumal die feuchten Rasselgeräusche beim Lungenödem am besten basal zu hören sind.

In der internationalen Konsensuskonferenz wurde die diffuse Präsenz von B-Linien auf verschiedene pulmonale Erkrankungen ausgeweitet:

- Lungenödem verschiedener Genese,
- interstitielle Pneumonie oder Pneumonitis,

- diffuse interstitielle Lungenparenchymerkrankungen (Lungenfibrose, exogen allergische Alveolitis, Sarkoidose, Asbestose usw.; [1]).

Diese Ausweitung der Indikation führt zu einer Reduktion der Spezifität. Nach Meinung des Autors sollte der B-Linien-Hype auf die Frage nach „sound of lung water" bzw. auf die Herzinsuffizi- 


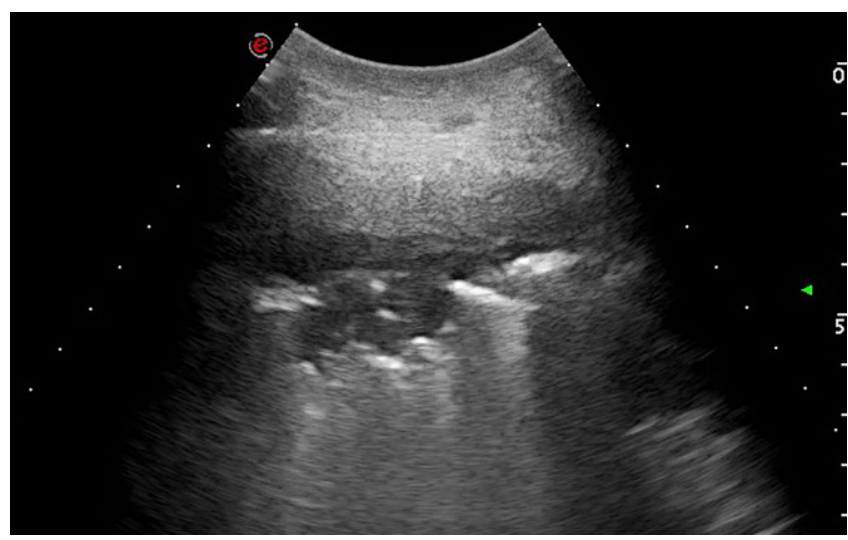

Abb. 4 \ Randständige Pneumonie mit Bronchoaerogramm: zackige Begrenzung, Kometenschweifartefakte und kleiner parapneumonischer Erguss

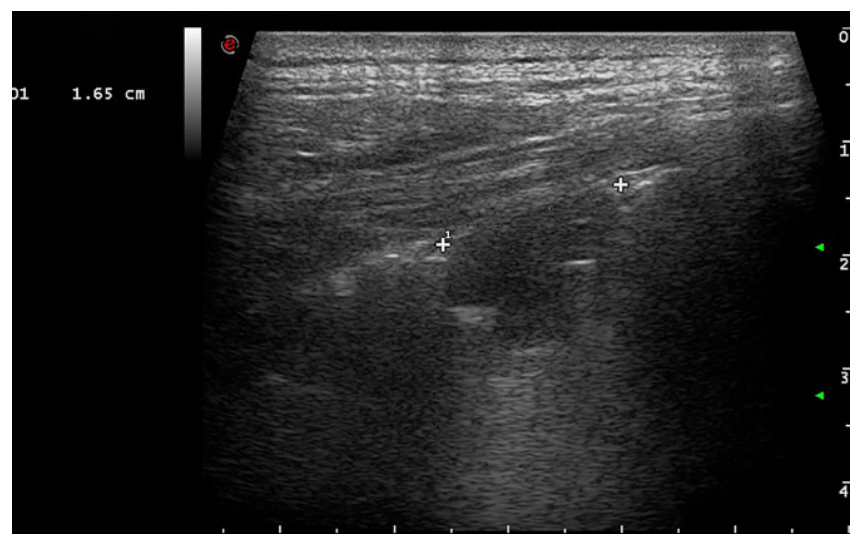

Abb. 5 - Sonographische Bild einer peripheren Lungenembolie enz mit semiquantitativer Verlaufsbeurteilung beschränkt und fokussiert werden [12-14]. Auch beim ARDS finden sich vermehrte B-Linien. Diese sind dabei jedoch unregelmäßig verteilt: zwischen Regionen mit vermehrten B-Linien zeigen sich Areale mit normaler Belüftung.

\section{Lungenkonsolidierungen}

Ätiologisch gesehen bestehen 4 Formen von Lungenkonsolidierungen, die sonographisch dargestellt werden können:

- inflammatorisch ( $\rightarrow$ Pneumonie),

- mechanisch ( $\rightarrow$ Atelektase),

- vaskulär ( $\rightarrow$ Lungenembolie),

- neoplastisch ( $\rightarrow$ Karzinom und Metastase).

Wenn auch selten ein Patient mit Lungenkarzinom wegen Schmerzen und Luftnot in die Notaufnahme kommt, sollen Neoplasien hier aus Platzgründen nicht weiter vertieft werden.

\section{Pneumonie}

Die häufigste Diagnose bei Atemnot in der Notaufnahme ist mit $35 \%$ eine Pneumonie [3]. Sonomorphologisch ist die bakterielle Pneumonie durch eine echoarme subpleurale Konsolidierung charakterisiert, in der sich ein dynamisches Bronchoaerogramm zeigt. Die Dynamik des Bronchoaerogramms ist zur Abgrenzung einer Obturationsatelektase essenziell (• Abb.4). Der Rand ist zackig begrenzt und zeigt Kome- tenschweifartefakte. Bedeutend ist eine kräftige reguläre Durchblutung insbesondere zur Differenzierung von Lungenembolien. Virus- und Pilzpneumonien sind meistens kleiner und zeigen weniger Bronchoaerogramme. Parapneumonische Pleuraergüsse werden in der LUS bei $55 \%$, im Röntgenthoraxbild zu $25 \%$ gesehen. Durch deren Überwachung können Pleuraempyeme rechtzeitig erkannt und therapiert werden. Im klinischen Verlauf korreliert die Wiederbelüftung der pneumonischen Lungenkonsolidierung mit der LUS besser als mit dem Röntgenthoraxbild, in dem noch länger interstitielle Restinfiltrationen nachweisbar sind, obwohl die Patienten wieder gesund sind $[15,16]$.

In 4 Metanalysen wurde die LUS mit der Computertomographie als Referenzmethode evaluiert. Dabei zeigt sich für LUS eine Sensitivität von $88-97 \%$ und eine Spezifität von 90-96\%. Die Thoraxröntgendiagnostik hingegen wies eine gepoolte Sensitivität von $77 \%$ und eine Spezifität von $91 \%$ auf. Daher sollte LUS die Thoraxröntgendiagnostik in der bildgebenden Diagnostik der ambulant erworbenen Pneumonie ersetzen [17].

\section{Atelektasen}

Bei beatmeten Patienten ist es schwieriger, sonographisch zwischen resorptiver Atelektase und einer Pneumonie zu unterscheiden. Dazu ist die klinische Gesamtschau inklusive Labordiagnostik erforderlich. Doch wurde gezeigt, dass solche Konsolidierungen mittels LUS gut nachweisbar sind und dass die Reventilation mit der Computertomographie korreliert [18]. Kompressionsatelektasen in voluminösen Pleuraergüssen zeigen eine typische Sonomorphologie: Sie sind schmal, zipfelmützenförmig, bewegen sich im Erguss wie eine winkende Hand und sind bei Inspiration teilweise wieder belüftet.

\section{Thromboembolie}

Mit Einführung der Mehrschichtspiralcomputertomographie (MSCT) hat die Inzidenz der Lungenembolie zugenommen, deren Mortalität konnte jedoch seit 3 Jahrzehnten nicht gesenkt werden [19, 20]. Da die klinischen Symptome unspezifisch sind, wird in der Notaufnahme zu selten daran gedacht oder diagnostisch zu langsam vorgegangen. Die klinische Wahrscheinlichkeit spielt wieder zunehmend eine entscheidende Rolle. Bei hoher klinischer Wahrscheinlichkeit sollte sofort eine adäquate Bildgebung erfolgen und kein D-Dimer-Test mehr durchgeführt werden.

Ein hämodynamisch instabiler Patient sollte bei Verdacht auf eine Lungenembolie sofort echokardiographiert und bei Zeichen der Rechtsherzbelastung sofort lysiert werden. Etwa die Hälfte der Pulmonalarterienembolien stammen aus den tiefen Beinvenen. Die 2-PunktKompressionssonographie der V. femoralis und der V. poplitea hat sich laut aktuellen Leitlinien ausreichend bewährt 
und kann rasch durchgeführt werden [21].

\section{》) Je nach Beschwerden und klinischem Status ist eine Triple- Mode-Sonographie sinnvoll}

Je nach Beschwerden und klinischem Status ist die Triple-Mode-Sonographie sinnvoll: Herz, Lunge und Beinvenen. Virchow hat schon vor 150 Jahren festgestellt, dass die Thromboembolie eine gemeinsame Erkrankung darstellt. Die beste Sensitivität zur Diagnostik der Lungenembolie hat die LUS [22]. Dabei zeigen sich relativ kleine echoarme Lungenkonsolidierungen ( $1-3 \mathrm{~cm}$ groß) mit pleuraler Basis (überwiegend triangulär, durchschnittlich 2,4 Läsionen pro Patient; - Abb. 5).

Zentral lässt sich in der Farb-Doppler-Sonographie keine Durchblutung nachweisen. In der Hälfte der Fälle zeigt sich ein kleiner lokaler oder basaler Pleuraerguss [23]. Metaanalysen zeigen für die alleinige LUS eine Sensitivität von $80-87 \%$ und eine Spezifität von $82-93 \%$ [24]. Eine Senkung der Mortalität kann erreicht werden, wenn möglichst zeitgerecht eine Triple-Ultraschalluntersuchung durchgeführt wird $[25,26]$.

\section{Fazit für die Praxis}

- Jeder Kliniker ist gefordert, bei Dyspnoe oder Thoraxschmerzen die Lungen-/Thoraxsonographie in Kombination mit der fokussierten Echokardiographie einzusetzen.

- Aktuell besteht die Möglichkeit, mit mobilen Geräten die Auskultation mit dem Stethoskop zu ergänzen oder gar zu ersetzen.

- Viele der beschriebenen Lungenerkrankungen in der Akutmedizin können rasch bildgebend diagnostiziert werden. Dies führt über eine frühzeitige Diagnose zu einer besseren Behandlung der Patienten und spart Ressourcen.

\section{Korrespondenzadresse}

Prof. Dr. Gebhard Mathis

Dr. Summer-Straße 3, 6830 Rankweil, Österreich gebhard.mathis@cable.vol.at

\section{Einhaltung ethischer Richtlinien}

Interessenkonflikt. G. Mathis gibt an, dass kein Interessenkonflikt besteht.

Für diesen Beitrag wurden vom Autor keine Studien an Menschen oder Tieren durchgeführt. Für die aufgeführten Studien gelten die jeweils dort angegebenen ethischen Richtlinien.

\section{Literatur}

1. Volpicelli G, Elbarbary M, Blaivas M et al (2012) International evidence-based recommendations for point-of-care lung ultrasound. Intensive Care Med 38:577-591

2. Kreuter M, Mathis G (2014) Die notfallmäßige Thoraxsonographie. Karger Kompass Pneumol Respiration 87:89-97

3. Zanobetti M, Scorpiniti M, Gigli Cet al (2017) Pointof-care ultrasonography for evaluation of acute Dyspnea in the ED. Chest 151:1295-1301

4. Lichtenstein D, Malbrain MLNG (2017) Lung ultrasound in the critically ill (LUCI): a translational discipline. Anaesthesiol Intensive Ther 49:430-436

5. Reuss J (2010) Sonography of the pleura. Ultraschall Med 31:8-22

6. Balik M, Plasil P, Waldauf P et al (2006) Ultrasound estimation of volume of pleural fluid in mechanically ventilated patients. Intensive Care Med 32:318-321

7. Teichgräber P, Hackbarth J (2018) Sonographic bedside quantifcation of pleural effusion compared to computed tomography volumetry in ICU patients. Ultrasound Int Open 4:E131-E135

8. Soldati G, Testa A, Silva F et al (2006) Chest ultrasonography in lung contusion. Chest 130:533-538

9. Volpicelli G (2011) Sonographic diagnosis of pneumothorax. Intensive Care Med 37:224-232

10. Gehmacher O, Kopf A, Mathis G et al (1997) Ist eine Pleuritis sonographisch darstellbar? Ultraschall Med 18:2014-2019

11. Lichtenstein D, Meziere G (1998) A lung ultrasound sign allowing bedside distinction between pulmonary edema and COPD: the comet-tai artifact. Intensive Care Med 24:1331-1334

12. Soldati G, Demi M, Inchingolo R, Smargiassi A, Demi $L$ (2016) On the physical basis of pulmonary sonographic interstitial syndrome. J Ultrasound Med 35:2075-2086

13. Dietrich CF, Mathis G, Blaivas M (2016) Lung B-line artefacts and their use. J Thorac Dis 8:1356-1365

14. Pivetta E, Baldassa FF, Masellis SS et al (2018) Sources of variability in the detection of B-lines using lung ultrasound. Ultrasound Med Biol 44:1212-1216

15. Gehmacher O, Mathis G, Kopf A et al (1995) Ultrasound imaging of pneumonia. Ultrasound MedBiol 21:1119-1122

16. Reissig A, Copetti R, Mathis G et al (2012) Lung ultrasound inthe diagnosis and follow-up of community-acquired pneumonia. A prospective multicentre diagnostic accuracy study. Chest 142:965-972

17. Pneumonie MG (2018) Ersetzt der Ultraschall das Thoraxröntgen? Praxis 107:1-5

18. Wang G, Ji X, Xu Y et al (2016) Lung ultrasound: a promising tool to monitor ventilator-associated pneumonia in critically ill patients. Crit Care 20:320-336

19. Burge AJ, Freeman KD, Klapper PJ, Haramati LB (2008) Increased diagnosis of pulmonary embolism without a corresponding decline in mortality during CTera. Clin Radiol 63:381-386

20. Öszu SS, Durmus ZG, Coskuner MB et al (2017) Does the incidence and mortality of pulmonary embolism change over the years? Turk Thorac J 18:78-81

21. Hach-Wunderle V, AMWF-guidelines ZC (2016) Thrombosis and pulmonary embolism. Vasa 45(Suppl.90):1-48

22. Nazerian P, VanniS, Volpicell G etal (2014) Accuracy of point-of-care multiorgan ultrasonography for the diagnosis of pulmonary embolism. Chest 145:950-957

23. Mathis G, Blank W, Reissig A et al (2005) Thoracic ultrasound for diagnosing pulmonary embolism. A prospective multicenter study of 352 patients. Chest 128:1531-1538

24. Jiang L, Ma Y, Zhao C et al (2015) Role of thransthoracic ultrasonography in the diagnosis of pulmonary embolism: a systematic review a metaanalysis. PLoSONE 10:e129909

25. Mathis G (2014) Thromboembolism in ultrasound: killing three birds with one stone. Chest 145:931-932

26. Zhu R, Ma XC (2017) Clinical value of ultrasonography in diagnosis of pulmonary embolism in critically ill patients. J TransI Intern Med 5:200-204 